\title{
The Ability of Children 18-36 Months to Distinguish between Pretending and Intentional Actions as Age Cognitive Development Indicator
}

\author{
Besjona Dede \\ PhD student, Department of Psychology and Pedagogy, \\ Faculty of Social Sciences University of Tirana. \\ besjonadede@gmail.com
}

\section{Doi:10.5901/jesr.2014.v4n6p37}

\begin{abstract}
Child development characteristic that appears at the age of 2 years old is engaging in symbolic plays action or sequences. The study included 18 children from 18 to 24 months and 37 children 25 to 36 months, that frequent public day care centers in Tirana (capital city of Albania). The protocol is applied to participants to estimate pretend and intentional behaviors. The purpose of the study is to explore the ability of children 18 to 36 months to distinguish intentional and pretend, as indicators of their cognitive skills development and the engagement in pretend play. The study is part of a more expansive research in time and purpose. Results of the current study show the tendency of children to distinguish the usage of pretending and intentional behavioral structures in accordance with the behavioral typology. This capability is most notable in the age 24-36 months who understand the pretend action from the intentional one. In conclusion, this study aims to provide a set of recommendations for parents, care givers and education specialists who work with children of this age including pretend elements in every day practices and activities. Such practices in day care institutions have been used rarely and spontaneously. The findings tell about the importance and necessity of using symbolic/pretend play in practices and curricula of educational institutions for early childhood with the aim of enhancing quality of care and education for this age group in a society where such practices do not have a tradition of usage.
\end{abstract}

Keywords: cognitive development, symbolic/pretend play, early childhood development, early childhood education.

\section{Introduction}

Play is the main activity during childhood. As a result of cognitive development in early childhood does appear the symbolic play elements. Likewise, the pretend play itself enables children cognitive skills development. In cognitive field children develop the symbolic reasoning. The purpose of the study is to explore the ability of children 18 to 36 months to distinguish between intentional and symbolic actions.

\subsection{Research question}

The research questions of the study are: Does children 18 to 36 months have the ability to distinguish actions as intentional or symbolic? 1 . Which is the relationship between early childhood age group and trying/pretending behavior that children exhibit?

\section{Which is the Gender Ratio in Pretending/ Trying Behavior in Different Age Groups in Early Childhood?}

This study is based on several studies of Rakoczy et.al; Lillard, 1994; Nichols \& Stich, 2000; Harris and Kavanaugh (1996).

"These studies provide clear evidence of the fact that children 3 years old and understand the structure within the intention of pretending behavior as a form of action that differs from other forms of "if" behavior. These data prove that even younger children can understand the elements of the alleged conduct. While data on children 2 years of age are still unclear ... " Rakoczy. H and Tomasello.M (2006). 


\subsection{Theoretical concepts}

The theoretical concepts used in this study are pretending play, cognitive development during early childhood, public day care institutions.

The concept "pretend play" in the literature is found as synonymous with imaginary play, symbolic play. In this study these concepts interchangeably refer to activities where objects were attributed symbolic action.

Thus, the symbolic or pretend play is a stage of playing that include children who are able to attribute symbolic action to the objects or people. The pretend play creates a world in which children can express their ideas and feelings about life (S J Goldstein \& Naglieri, 2011). The pretend or imaginary play helps children develop thinking skills or problem solving. Cognitive development as part of the overall development of the individual displays certain characteristics in different stages of development. During early childhood cognitive development involves the use of problem solving skills and abstract thinking. The ability to use symbolic thinking is a mental skill level high (Robinson, 2008). During the ages of 2 and 3 year old children in cognitive terms have the ability to fulfill certain tasks. At the age of 2 they can find objects even when they are covered, choose objects based on color and shape. So at the age of three they can play with toys, "if" play with dolls, animals and people (Robinson 2008). Children during early childhood already do understand pretend behavior as a specific form of behavior with a goal, but they still do not understand the structure of epistemic pretend (Lilliard, 1998). Institution of child care (0-3 years old) refers to special public institutions for the welfare and education of children aged 0-3 years. On the conceptual relationship between symbolic play and cognitive development Berger (2006) argues that symbolic play requires the ability to transform into symbolic objects and actions, followed by dialogue and negotiation, involves taking roles and improvisation. Many cognitive strategies symbolic appear during the play. Thus, the elements of the complex imaginary play develop parallel with the appearance of pretending behavior. Because of the first steps of the mental representation development and symbolism in approximately the same age (usually at 1 and 2 years old), there is a strong conceptual linkage between these concepts ( Harris and Kavanaugh (1996) cited from Tomasello and Rakoczy).

\subsection{Theory and study}

This study is based on the classical theory of Piaget on cognitive development in early childhood, focusing on the use of symbolic functions. According Piaget, the cognitive development goes through several stages. Children 2-7 years of age are at the stage of pre-operational thinking. During this stage, children learn symbols representing objects, play with dolls, develop imagination, imitate the actions of adults (Orhan, 2005). The main principle is related to the child, not as a being that simply mimics, but as an organism assimilates, selects and develops in accordance with the structure itself (J. Piaget, 1976). Studies in recent years on the development of child support the idea of association between cognitive development and symbolic play during early childhood. Thus, Harris and Kavanaugh, after realizing some experiments proved that around the age of 2 years children begin to understand and follow the pretend actions by presenting them in an pretend scenario. As related to cognitive ability, Liliard (1998) has noted that pretend play involves negotiation between parties with different views, the representation of objects in two ways (real and pretend), role play, all actions that suggest the children involved in these activities have mental representation skills. In the trying/ pretend study (Rakoczy \& Tomasello 2006) children 22 months presented the same general patterns of response, although in an easier way: Generally these children responded to the two distinct types of models, which revealed the fact that each type of conclusive answers was performed significantly more often in the same conditions compared with the opposite conditions.

Rakoczy, Tomasello and Striano (2004, cited by Rakoczy f.557 \& Tomasello 2006) argued that young children already understand the pretend as a specific form of action-making as though they do not yet understand the epistemic structure of claim.

Usually the symbolic play appears during the second year of life. Frequency of occurrence is greater during preschool and begins to fade over the age of 6 years (Fein, 1981 p. 21 quoted by Kelly, et.al. 2011).

Symbolic play as often determined by deliberate splitting of reality in the children play when they behave "as if something is real when it is not (Leslie, 1987, f.413 cited by Kelly, et.al. 2011). According to Leslie (1987, cited by Kelly, et.al. 2011)pretend involves three essential forms: substitution of one object with another, attributing the features missing or not truth and imagination on the missing objects. However, it is generally accepted that this description is too narrow and that the game may involve symbolic attribution of animism (Jefree \& MocConkey, 1976; Lillard, 1993, Watson and Fischer, 1977, cited by Kelly, et.al. 2011) and role plays (Brown, Prescott, Richards \& Patterson, 1997, cited by Kelly, 
et.al. 2011).

\section{Methodology}

\subsection{Participants}

The sample consisted of 37 children 18 -24 months old and 18 children 25-36 months old; of which 25 girls and 30 boys. The children selected attend public day care and education institutions (crèches in Tirana city). The Groups of children were selected at random at 3 day care centers. The selection criteria were age and gender. The selection ratio of the age and gender aimed to respect gender and age ratios in the general population of children that frequent the day centers in Tirana. The selection of institutions have all been conducted based on criteria of homogeneity of social factors.

Age group' participants

\begin{tabular}{|cc|c|c|c|c|}
\hline & & Frequency & Percent & Valid Percent & Cumulative Percent \\
\hline \multirow{3}{*}{ Valid } & 18-24 months & 18 & 32.7 & 32.7 & 32.7 \\
& 25-36 months & 37 & 67.3 & 67.3 & 100.0 \\
& Total & 55 & 100.0 & 100.0 & \\
\hline
\end{tabular}

Gender' participants

\begin{tabular}{|cc|c|c|c|c|}
\hline & & Frequency & Percent & Valid Percent & Cumulative Percent \\
\hline \multirow{3}{*}{ Valid } & Girls & 25 & 45.5 & 45.5 & 45.5 \\
& Boys & 30 & 54.5 & 54.5 & 100.0 \\
& Total & 55 & 100.0 & 100.0 & \\
\hline
\end{tabular}

\subsection{Application}

There was applied a model based on the protocol used by Hannes Rakoczy and Michael Tomasello (2006) by a trained specialist. Activities and games used in this procedure were selected from the education program that is implemented in public kindergartens based on group age. There were presented two models of action: if it was eating / trying to eat; pour pretending / trying to pour. Each child was presented two action models of trying and two action models of pretending by the same theme. The order of operations and the definition of objects of the same subject to trying and pretending actions change from case to case. The order of topics within the same sequence was the same. In the beginning the tester specialist and child played freely, the tester did some simple actions, two pretending actions with an object whatever, two pretend actions and asks the child to do the same action. Then realized the first testing session, which consists of two models of actions. The procedure followed by the second session of the trying and pretend the extra phase and the second testing session. During the application protocol a specialist based on coding conducted the behavior classification.

\subsection{Procedures and preparatory actions}

Simple actions preparatory of pretend and trying (1) dig a whole (with a new object, (2) behave if makes a call (with the same object) (3) trying to make music with piano children (by pressing a button that does not work) 2 . The first trying and pretending section with additional action: (1) behave if someone is brushing the teeth, (2) behave if it is making a shower (3) attempting to open a container (using pliers), (4) trying to open a second container (then using pliers), (5) act if it is drinking and as it is opening a bottle of fruit juice, (6) cutting some dough with pliers, (7) behave if it is cooking something.3. session of the first test (for half of the children the pretending behavior, the other half the trying behavior) 4 . The second trying and pretend session with additional action, (1) behave if someone combs the hair, (2) trying to open a box, (3) behave it is washing something, (4) tries to write (with a pen that has a lid) .5. The second testing session (for half of the children the pretending behavior, the other half the trying behavior).

Each coding behavior was classified into one of five categories: "inferential pretend" when the child performs an action in accordance with the thematic contender, that goes beyond what the tester performs; "simple pretend" when the child performs what seems like a clear contender. Conversely, responses were coded as "inferential trying" when the 
child discovers from an action / word recognition that the goal was to implement a successful action or as "simple trying" when the child perform something that looks like a clear example of a trying behavior. Finally, the category "unclear" answers that do not meet the above categories.

\subsection{Validity and ethical principles.}

The using standard procedures has minimize subjectivity in applying the protocol from the testers and in data interpretation. The procedure is carried out by a tester/specialist who is instructed to use the same standards applicable to each behavioral sequence of each child. The tester is supervised. The behavioral coding is performed by an appraiser who is instructed and trained to follow the same behavior as indicators of ability. To increase the validity of coding is used a double check procedure. The institutional permission and the informed consent is obtained in advance by the institutions and parents. During the study the ethical and moral principles have been respected. Confidentiality was respected during the work with children.

\section{Results}

\subsection{Which is the relationship between early childhood age group and trying/pretending behavior that children exhibit?}

Age group * children behavior M1 Crosstabulation

\begin{tabular}{|cc|c|c|c|c|c|}
\hline & \multicolumn{4}{|c|}{ Children behavior M1 } & \multirow{2}{*}{ Total } \\
\cline { 3 - 7 } & & Simple trying & Simple pretend & Inferential trying & Inferential pretend & \\
\hline \multirow{2}{*}{ Age group } & $18-24$ months & 9 & 6 & 1 & 2 & 18 \\
& $25-36$ months & 3 & 9 & 8 & 17 & 37 \\
& Total & 12 & 15 & 9 & 19 & 55 \\
\hline
\end{tabular}

In the age group of 18-24 months the most display behavior in children has been the simple trying, then the simple pretend and only 3 children have shown second behavior. So the dominant level of age group behavior has been the first level behavior. While children 24-36 months have shown more second level behavior as inferential trying and inferential pretend. This fact supports and data study according to the procedure used and cited in other studies in the literature

\subsection{Which is the gender ratio in pretending/ trying behavior in different age groups in early childhood?}

Gender * children behavior M1 * age group Crosstabulation

\begin{tabular}{|c|c|c|c|c|c|}
\hline \multirow{2}{*}{ Age group } & \multicolumn{4}{|c|}{ Children behavior M1 } & \multirow{2}{*}{ Total } \\
\hline & Simple trying & Simple pretend & Inferential trying & Inferential pretend & \\
\hline \multirow{3}{*}{ 18-24 months } & 2 & 4 & 1 & 0 & 7 \\
\hline & 7 & 2 & 0 & 2 & 11 \\
\hline & 9 & 6 & 1 & 2 & 18 \\
\hline \multirow{3}{*}{ 25-36 months } & 2 & 4 & 2 & 10 & 18 \\
\hline & 1 & 5 & 6 & 7 & 19 \\
\hline & 3 & 9 & 8 & 17 & 37 \\
\hline \multirow{3}{*}{ Total } & 4 & 8 & 3 & 10 & 25 \\
\hline & 8 & 7 & 6 & 9 & 30 \\
\hline & 12 & 15 & 9 & 19 & 55 \\
\hline
\end{tabular}

From the collection and processing of data it shows that in the age group 18-24 months 2 girls and 7 boys have shown simple tying models presented, 4 girls and 2 boys have shown simple pretend, 1 girl and no boy has shown inferential trying, 2 boys and no girl has shown inferential pretending. So the largest number of children has shown simple trying compared with all kinds of behavior. While the largest number of girls has shown simple pretending that is a behavior typical for their age group, so the first behavioral level. The large number of boys have shown simple trying, while the results of boys and girls have had differences in behavior between the occurrence of the first level as simple trying and pretend and low difference in the behavior of the second level as the inferential trying and inferential pretend. 
From the collection and processing of data it shows that in the age group 25 to 36 months the number of girls who exhibit simple trying behavior is not much different from the number of boys who display this behavior; The same report is notable in simple trying behavior but here the number og boys is bigger than the number of girls. On the second level of behavior, defined as usual for age differences between boys and girls is high; boys showed more inferential trying behavior while girls showed more inferential pretending behavior.

From the results it is notable that there are significant gender differences on the level of behavior that is typical and the difference is smaller for the level of uncommon behavior for the age group.

\section{Discussion}

According to the research issues studied in this article, concrete results have shown that children 18 to 36 months do have the ability to distinguish trying actions with symbolic ones, displayed progressively during growth. Children 25-36 months have more notable abilities to distinguish between trying and pretending actions under the respective stimulation models, behavioral models and represent the second level, able to manipulate objects or simple action levels more complicated in pretend models; children 18-24 months understand the intention of the actions and follow the models presented, but find it difficult to improvise beyond the applied model featuring only first-level behaviors, and attempt simple pretend. In a more detailed comparison between the reactions compatible and not compatible after the presentation of each model, children presented more compatible answers than not compatible model after the presentation. Tracking model is associated with other factors of children development and the environment. Although it wasn't the purpose of this data collecting research data there were highlighted factors and conditions that affect the appearance of behaviors children 18-36 months. These tracks will be studied in future studies to better analyze the phenomenon. The current study is descriptive and aims to highlight the linkage between the variables age group, gender and behavior exhibited by children as an outcome that indicates the mutual influence of symbolic play in cognitive development and cognitive skills development in the performance of symbolic play in early childhood.

\section{Recommendations}

The study results are clear about the role of play in cognitive development during early childhood. The game has a symbolic role in the lifestyle during early childhood and its influence extends throughout the first years of school and later on. Policymakers, education specialists, parents, educators, caregivers need to understand the relationship between play and the development of cognitive skills, particularly skills to distinguish those trying and pretending actions in the first and second level; to influence the inclusion of symbolic play in practices and curricula of educational institutions for early childhood, with the aim of enhancing quality of care and education for this age group in a society where such practices do not have a tradition of usage.

\section{References}

S Goldstein \& J Naglieri, Encyclopedia of Child Behavior and Development, Springer Science+Business Media LLC, 2011, f. 522-523.

A Lillard, Playing with a theory of mind, In O. N. Saracho \& B. Spodek Multiple perspectives on play in early childhood, Albany: State University of Neë York Press, $1998 \mathrm{f}$. 11-33.

Z Orhani, Cognitive Psychology , Ada, 2005, f.61, 262-263.

R Shelov and R Hannermann, Caring for your baby and young child: Birth to 5, American Academy of Pediatrics, 2004 www.cdc.gov/actearly

D Bergen, The Role of Pretend Play in Children's Cognitive Development, ERIC Clearinghouse on Elementary and Early Childhood Education Champaign IL Miami University, Volume 4 Number 1, 2002.

M Robinson, Child Development 0-8, A Journey through the Early Years, Open University Press, NY, 2008, f.157

J Piaget, The child conception of the world, Littlefield, Adams and CO, Totoëa New Yersey, 1976, f.14

S Johnson, The nature of cognitive development, Trends in Cognitive Sciences, Vol.7, No.3, Mars 2003 f.102-104.

H Rakoczy and M Tomasello, Two-year-olds grasp the intentional structure of pretense acts, Developmental Science 9:6 (2006), f. 557564.

Kelly, R., Hammond, S., Dissannayake, Ch., Ihsen, E., The relationship between symbolic play and executive functions in young children, Australiasia Journal of Early Childhood, pp 21-28; 
ISSN 2239-978X

ISSN 2240-0524
Journal of Educational and Social Research MCSER Publishing, Rome-Italy
Vol. 4 No. 6 September 2014 